\title{
Risk of cardio-nephro-metabolic disease from NAFLD to MAFLD: fact or fiction?
}

\author{
Ramzi Hassouneh ${ }^{1}$, Mohammad Shadab Siddiqui ${ }^{1}$, Chandra Bhati ${ }^{2}$ \\ 'Division of Gastroenterology and Hepatology, Virginia Commonwealth University, Richmond, VA 23298, USA. \\ ${ }^{2}$ Division of Transplant Surgery, Virginia Commonwealth University, Richmond, VA 23298, USA.
}

Correspondence to: Dr. Chandra Bhati, Division of Transplant Surgery, Virginia Commonwealth University, 1250 E Marshall St, Richmond, VA 23298, USA. E-mail: chandra.bhati@vcuhealth.org

How to cite this article: Hassouneh R, Siddiqui MS, Bhati C. Risk of cardio-nephro-metabolic disease from NAFLD to MAFLD: fact or fiction? Metab Target Organ Damage 2021;1:4. https://dx.doi.org/10.20517/mtod.2021.07

Received: 24 May 2021 First Decision: 29 Jul 2021 Revised: 30 Jul 2021 Accepted: 10 Aug 2021 Available online: 17 Aug 2021

Academic Editor: Amedeo Lonardo Copy Editor: Yue-Yue Zhang Production Editor: Yue-Yue Zhang

\begin{abstract}
Nonalcoholic fatty liver disease (NAFLD) is emerging as the most common etiology for chronic liver disease. Despite this, our understanding of this illness is lacking. The previous paradigm is that central adiposity, hyperlipidemia, hypertension, and insulin resistance, also known as metabolic syndrome, lead to NAFLD, and this relationship is unidirectional. However, recent evidence clearly shows that the clinical burden of this illness extends well beyond liver-related morbidity and mortality and is associated with multiple extrahepatic complications, particularly metabolic consequences. Due to this, the professional consensus has proposed using the term metabolic associated fatty liver disease (MAFLD) to more accurately reflect pathogenesis and help in patient stratification for management. This review discusses the shared pathophysiological mechanisms that link these diseases and how this can be leveraged to prevent these complications in individuals with NAFLD/MAFLD.
\end{abstract}

Keywords: Nonalcoholic fatty liver disease, metabolic associated fatty liver disease, cardiovascular disease, diabetes, insulin resistance, chronic kidney disease

\section{INTRODUCTION}

Nonalcoholic fatty liver disease (NAFLD) encompasses a spectrum of liver diseases defined by the presence of hepatic fat accumulation in the absence of secondary causes of liver disease, such as significant alcohol 
use $^{[1,2]}$. Early-stage NAFLD, also known as non-alcoholic fatty liver, is characterized by isolated hepatic steatosis $^{[1,2]}$. As the disease progresses, isolated hepatic steatosis leads to inflammation and hepatocyte injury [non-alcoholic steatohepatitis (NASH)], fibrosis (NASH cirrhosis), and hepatocellular carcinoma ${ }^{[1,2]}$.

NAFLD is the most common chronic liver disease worldwide ${ }^{[3]}$. The burden of NAFLD on society continues to increase as a direct result of changes in dietary habits and sedentary lifestyles ${ }^{[3-5]}$. Recent estimates suggest a prevalence of up to $45 \%$ in the United States and $25 \%$ worldwide ${ }^{[3-5]}$. The prevalence of NAFLD in obese and diabetic individuals is as high as $75 \%$, in contrast to a prevalence of $15 \%-30 \%$ in the general population ${ }^{[3-5]}$. NAFLD was previously thought to be the hepatic manifestation of metabolic syndrome, as is suggested by its increased prevalence in those with obesity and type 2 diabetes mellitus (T2DM); however, recent data suggest that the impact of NAFLD is not limited to liver-related mortality and outcomes, but rather is a multi-system disease that can lead to multiple complications including coronary artery disease and cardiomyopathies, chronic kidney disease (CKD), and insulin resistance ${ }^{[6]}$.

Due to these changes in our understanding of NAFLD, Eslam et al. ${ }^{[7]}$ called for consensus to address the nomenclature of NAFLD so that it can more accurately reflect pathogenesis and help in patient stratification for management. After applying a carefully designed Delphi method, a representative panel of experts suggested metabolic associated fatty liver disease (MAFLD) as a more appropriate overarching term [Table 1].

\section{NAFLD/MAFLD AND INSULIN RESISTANCE}

NAFLD and T2DM often co-exist as they share many common risk factors, particularly obesity and insulin resistance. In fact, recently proposed diagnostic criteria for MAFLD recommends the following definition: detection of steatosis with one of the different modalities (imaging, blood biomarker, or histology) with the presence of one of three criteria, overweight or obesity, T2DM, or evidence of metabolic abnormalities ${ }^{[7]}$.

A recent meta-analysis of 19 studies with about 300,000 patients estimated that individuals with NAFLD had a 2 -fold higher risk of developing T2DM and a 5 -fold increase in the presence of advanced fibrosis ${ }^{[8]}$. In addition, one study examined that the risk of developing T2DM in patients with biopsy-proven NAFLD and found that approximately $80 \%$ of patients developed T2DM within 14 years $^{[9]}$.

The relationship between NAFLD and T2DM is quite complex as insulin resistance is associated with both intrahepatic and extrahepatic lipid accumulation and promotes liver injury and fibrosis. Under normal physiologic conditions, insulin suppresses hepatic glucose production. However, when intrahepatic fat accumulates, this physiological response is blunted as a result of insulin resistance. This results in poorer glycemic control in patients with both T2DM and NAFLD compared to patients with only T2DM ${ }^{[6]}$.

Insulin resistance and elevated blood glucose lead to many downstream effects and result in the many extrahepatic manifestations observed with NAFLD; however, the exact mechanism is yet to be elucidated. Multiple chemical mediators have been identified, some that have also been implicated in the development of cardiovascular disease (CVD) and CKD, including interleukin 6 (IL-6), tumor necrosis factor-alpha (TNF- $\alpha$ ), ceramides, diacylglycerols, gut microbiome dysbiosis, and mitochondrial dysfunction. Serum levels of adiponectin, an adipocytokine that protects against insulin resistance by promoting fatty acid oxidation and glucose utilization, were found to be reduced in patients with NAFLD compared to their control cohorts ${ }^{[10,11]}$. A low level of adiponectin was associated with higher levels of low-density lipoproteins and triglycerides and lower levels of high-density lipoproteins ${ }^{[12,13]}$. Elevations in lipid metabolites, particularly ceramides and diacylglycerols, have been found to occur in models of NAFLD and have been 
Table 1. Comparison of diagnostic criteria for NAFLD and MAFLD

\begin{tabular}{ll}
\hline NAFLD & MAFLD \\
\hline Diagnosis requires the following: & Must have hepatic steatosis (imaging or histology) AND one of the \\
& following: \\
(1) Evidence of hepatic steatosis (imaging or histology) AND & (1) Overweight (BMI $>25 \mathrm{~kg} / \mathrm{m}^{2}$ in Caucasians or $>23 \mathrm{~kg} / \mathrm{m}^{2}$ in Asians) \\
(2) Absence of secondary causes of liver disease (alcohol & (2) Normal weight $\left(\mathrm{BMI}<25 \mathrm{~kg} / \mathrm{m}^{2}\right.$ in Caucasians or $<23 \mathrm{~kg} / \mathrm{m}^{2}$ in \\
consumption, hereditary disorder, medication) & Asians) AND at least 2 of the following: \\
& (a) Waist circumference $>102 / 88 \mathrm{~cm}$ in Caucasians or $>90 / 80 \mathrm{~cm}$ in \\
& Asians \\
& (b) Blood pressure $>130 / 85 \mathrm{mmHg}$ or on anti-hypertensive medication \\
& (c) Triglycerides $>150 \mathrm{mg} / \mathrm{dL}$ or on treatment \\
& (d) HDL-cholesterol $<40 \mathrm{mg} / \mathrm{dL}$ for men or $<50 \mathrm{mg} / \mathrm{dL}$ for women or \\
& on treatment \\
& (e) Prediabetes \\
& (f) Insulin resistance $\mathrm{Score}>2.5$ (via homeostasis model assessment) \\
& (g) High-sensitivity C-reactive protein $>2 \mathrm{mg} / \mathrm{L}$ \\
& (3) Type 2 diabetes mellitus \\
\hline
\end{tabular}

NAFLD: Nonalcoholic fatty liver disease; MAFLD: metabolic associated fatty liver disease.

linked to insulin resistance ${ }^{[14]}$. Gut microbiota is emerging as an important mediator in the natural progression of T2DM in patients with NAFLD ${ }^{[15]}$. The gut microbiota in NAFLD is often in a state of dysbiosis with an increase of deleterious microorganisms in relation to beneficial ones. Studies have shown that restoration of gut microbiota in patients with NAFLD with treatment with antibiotics, probiotics, or fecal microbiota transplantation led to an increase in insulin sensitivity ${ }^{[16,17]}$. Mitochondrial dysfunction is commonly seen in NAFLD, likely due to excessive beta-oxidation of free fatty acids in the early stages of the disease, which leads to excess production of reactive oxygen species ${ }^{[18]}$. As NAFLD progresses, fatty acid beta-oxidation is downregulated, and lipotoxic intermediates accumulate, which may directly impact insulin signaling ${ }^{[19]}$.

It appears that the relationship between T2DM and NAFLD is bi-directional; not only does T2DM promote NAFLD progression to cirrhosis, increase all-cause and liver-related mortality, but NAFLD also leads to insulin resistance and poor glycemic control ${ }^{[20]}$. In addition, microvascular complications, including retinopathy and nephropathy, are more prevalent in individuals with T2DM and NAFLD than those with T2DM alone, and this relationship was independent of traditional confounding risk factors ${ }^{[2,22]}$. A study of individuals with NAFLD and type 1 diabetes demonstrated the same findings, suggesting that the common mechanism may be the release of pro-inflammatory mediators such as advanced glycated end-products, reactive oxygen species, TNF- $\alpha$, and transforming growth factor-beta ${ }^{[23,24]}$.

Due to the similar pathophysiologic mechanisms of NAFLD and T2DM, several anti-diabetic drugs have been trialed in patients with NAFLD. Metformin has been shown to be beneficial in patients with NAFLD by decreasing the amount of fat deposition in the liver and improving metabolic parameters, including the normalization of serum levels of aminotransferases ${ }^{[25,26]}$. A prospective trial on 42 NAFLD patients without T2DM demonstrated that metformin and dieting led to significant reductions in hepatic steatosis and fibrosis after 5 months, compared to patients treated with diet alone ${ }^{[27]}$. Interestingly, a meta-analysis of 17 randomized controlled trials showed that up to 12 months of metformin and lifestyle intervention did not improve liver histology or aminotransferases in patients with both NAFLD and T2DM ${ }^{[28]}$. Another promising class of drugs includes thiazolidinediones (TZD), which increase insulin sensitivity by activating peroxisome proliferator-activated receptors. Multiple randomized controlled trials have evaluated the efficacy of TZDs and demonstrated that these drugs provide a beneficial effect on lobular inflammation. However, their effect on steatosis and fibrosis is unclear ${ }^{[29-31]}$. Newer drugs such as the glucagon-like peptide- 
1 analogues have emerged as attractive therapeutics in patients with $\mathrm{NAFLD}^{[32]}$. After 48 weeks of treatment with liraglutide, patients with biopsy-proven NAFLD showed significant improvement in steatosis and hepatocyte ballooning; however, no significant differences were seen in regard to lobular inflammation and fibrosis-4 score ${ }^{[33]}$. A recent study showed that patients with NASH treated with semaglutide resulted in a significantly higher percentage of patients with resolution of NASH compared to placebo. However, the trial did not show a significant difference between groups in the percentage of patients with an improvement in the fibrosis stage ${ }^{[34]}$. A meta-analysis of 11 randomized controlled trials showed that use of GLP-1 agonists (including liraglutide, exenatide, dulaglutide, or semaglutide) when compared to placebo or reference therapy, resulted in significant reductions in the absolute percentage of hepatic adipose content on magnetic resonance imaging and serum enzymes, in addition to the histological resolution of $\mathrm{NASH}^{[35]}$.

Based on this data, it is beneficial to screen patients with NAFLD for T2DM with yearly serum glycated hemoglobin (HgbA1c) and encourage weight loss and lifestyle changes, including dietary avoidance of fructose. In addition, due to their increased risk of complications, patients with NAFLD and established T2DM should be regularly screened for both microvascular and macrovascular changes.

\section{NAFLD/MAFLD AND RENAL DISEASE}

$\mathrm{CKD}$, defined as loss of kidney function as estimated by glomerular filtrate rate (GFR) less than 60 $\mathrm{mL} / \mathrm{min} / 1.73 \mathrm{~m}^{2}$ or presence of overt proteinuria, is present in up to $50 \%$ of individuals with $\mathrm{NAFLD}^{[23]}$. The most common causes of CKD are hypertension and T2DM, which are closely intertwined with metabolic syndrome, and have a strong association with NAFLD as well. However, studies have shown that NAFLD may accelerate the development and progression of CKD, regardless of hypertension or diabetes status ${ }^{[3,3,3]}$. The increased risk of CKD in NAFLD is further highlighted by the fact that NASH cirrhosis is the leading and most rapidly growing indication for simultaneous liver and kidney transplantation ${ }^{[38]}$. Individuals who receive a liver transplant for NASH cirrhosis remain at risk for developing CKD during the post-transplant follow-up ${ }^{[39,40]}$.

A meta-analysis of over 20 studies with more than 60,000 patients evaluated the prevalence and incidence of $\mathrm{CKD}$ in patients with fatty liver, NASH, and advanced fibrosis and found that incidence of CKD increased with more advanced NAFLD, suggesting that the severity of NAFLD is linked to $\mathrm{CKD}^{[37]}$. In addition, a randomized controlled trial with patients with biopsy-proven NASH demonstrated that GFR improved after one year of lifestyle intervention and resolution of NASH, while no improvement in GFR was observed in individuals that did not have lifestyle intervention ${ }^{[41]}$.

The pathological mechanisms linking NAFLD and CKD are thought to include dysregulation of angiotensin-converting enzyme (ACE)-2, impaired antioxidant mechanisms, hyperuricemia, and gut microbiota dysregulation ${ }^{[42]}$. The renin-angiotensin-aldosterone system is upregulated in obese patients due to adipocyte production of ACE-1 ${ }^{[43]}$. ACE-1 is responsible for converting the inactive hormone angiotensin-2 to the active hormone angiotensin-2. Angiotensin-2 has detrimental effects on both the liver and kidney by promoting insulin resistance, lipid production and deposition, mitochondrial oxidative stress, inflammation, and fibrosis ${ }^{[44,45]}$. Under normal physiologic conditions, ACE-2 degrades active angiotensin-2 to inactive angiotensin-1, which limits its activity. Although not studied in humans, experimental animal models of NASH showed that ACE-2 levels were significantly depressed ${ }^{[46]}$.

Multiple studies have shown that increased intake of dietary fructose was associated with worsening liver fibrosis and renal function ${ }^{[47,48]}$. This mechanism is thought to be secondary to fructose metabolism in hepatocytes, which leads to adenosine triphosphate depletion and consequently an increase in adenosine 
monophosphate conversion to uric acid. Hyperuricemia is associated with mitochondrial oxidative stress in hepatocytes and inhibits nitric oxide generation in endothelial cells, which is detrimental to the kidney ${ }^{[49]}$.

The relationship between CKD and NAFLD appears to be bi-directional. CKD leads to the build-up of uremic toxic metabolites, which can induce gut microbiota dysbiosis, which in turn promotes NAFLD. In particular, ammonia and ammonium hydroxide cause alterations in intestinal tight junctions and provide substrates to urea metabolizing microbiota ${ }^{[5,51]}$. These alterations subsequently allow the passage of lipopolysaccharides and activate inflammasome pathways in the liver, triggering liver injury and progression of NAFLD. Resolution of urea-induced disruption of colonic epithelial tight junctions by using oral activated charcoal in mouse models of CKD attenuated associated endotoxemia, oxidative stress, and inflammation ${ }^{[52]}$.

These findings suggest that a strong association exists between NAFLD and CKD, including a relationship that corresponds with NAFLD severity. Therefore, the management of CKD and its sequelae should be addressed early and incorporated in the care of patients with NAFLD. Given this relationship, it is likely that improvement in NAFLD may improve CKD, and the management of CKD may ameliorate the clinical outcomes in NAFLD.

\section{NAFLD/MAFLD AND CARDIOVASCULAR DISEASE}

CVD accounts for approximately $20 \%$ of death in individuals with NAFLD, making it the leading cause of mortality in this population ${ }^{[53]}$. NAFLD has been associated with traditional cardiometabolic risk factors, including obesity, hyperlipidemia, hypertension, and insulin resistance; however, the relationship between NAFLD and CVD is not entirely clear. Recent evidence appears to suggest that NAFLD, independent of the above risk factors, leads to additional risk for premature $\mathrm{CVD}^{[5,55]}$. In addition, new studies have identified non-traditional risk factors, such as pro-inflammatory cytokines (e.g., IL-6, TNF- $\alpha$ ), gamma-glutamyl transferase (GGT), fibrinogen, plasminogen, vascular adhesion molecules, C-reactive protein, adiponectin, and uric acid, which may link these two processes ${ }^{[56-58]}$. Interestingly, many of these molecules that link CVD and NAFLD are synthesized in the liver. A better understanding of this relationship is important, as it would allow us to target therapy for the treatment of liver disease to further ameliorate the risk of CVD.

NAFLD is linked with increased subclinical atherosclerosis and increased coronary artery calcium (CAC) deposition, as well as CVD itself ${ }^{[59]}$. Studies have demonstrated a link between NAFLD and increased carotid intima-media thickness, a well-validated tool for assessing atherosclerosis in asymptomatic patients that independently predicts CVD events ${ }^{[60-63]}$. A meta-analysis demonstrated that NAFLD was independently associated with a 3.7-fold higher likelihood of having carotid plaques ${ }^{[64]}$. Arterial stiffness, measured using the cardio-ankle vascular index, is closely associated with coronary atherosclerosis and stroke and was found to be increased in patients with NAFLD ${ }^{[65-68]}$. Computed tomography-based quantification of coronary atherosclerosis or CAC score has recently been demonstrated to be perhaps the most reliable noninvasive biomarker for cardiovascular health ${ }^{[69]}$. CAC scores in individuals with ultrasound confirmed NAFLD was found to be elevated compared to their control counterparts ${ }^{[70]}$. A longitudinal study demonstrated that NAFLD plays a role in the initial development of coronary artery calcification without any baseline calcification. As seen by liver ultrasound, the severity of NAFLD is positively associated with $\mathrm{CAC}$ in a dose-dependent fashion ${ }^{[71]}$. Another study that screened patients scheduled to undergo coronary angiogram with abdominal ultrasounds observes that fatty liver was associated with significant coronary stenosis independent of other metabolic factors ${ }^{[72]}$. 
Epidemiological studies clearly establish an association between NAFLD and the risk of cardiovascular events. In a large study of almost 20,000 patients without known liver disease, ultrasound-detected NAFLD was associated with an elevated 10-year risk of CVD events as estimated by the Framingham risk score, independent of traditional risk factors typically associated with metabolic syndrome ${ }^{[73]}$. Prospective studies have shown that the risk of CVD events is correlated with the extent of biopsy-proven fibrosis of NAFLD rather than other histological factors ${ }^{[74]}$. When adjusted for traditional CVD risk factors, fibrosis stage was the strongest predictor for CVD-related mortality in patients with NAFLD even after 33 years of follow$\mathrm{up}^{[74]}$. Furthermore, it was observed that patients with NAFLD and advanced fibrosis had CVD events predominantly, while those with NAFLD cirrhosis had liver-related events predominantly over a mean follow-up of 5.5 years $^{[75]}$.

As the primary etiology of cirrhosis prior to liver transplant, NASH has been shown to infer a higher longterm risk for CVD mortality compared to those transplanted for other causes of cirrhosis ${ }^{[76]}$. Furthermore, the long-term risk was affected, and CVD risk within one year of liver transplantation was increased in patients transplanted for NASH cirrhosis compared to patients transplanted for alcohol-induced cirrhosis $^{[77,78]}$.

One proposed mechanism linking NAFLD and CVD is serum GGT. GGT is frequently elevated in patients with NAFLD, and it has been reported to be associated with cardiovascular death, as well as increased nonfatal cardiovascular events, even in individuals with low to moderate cardiovascular risk ${ }^{[79]}$. In addition, Serum GGT undergoes redox reactions and is deposited in atherosclerotic plaques, likely leading to their progression $^{[80]}$. In fact, serum GGT was validated as a potential biomarker for future cardiovascular disease risk $^{[81]}$.

The effect of NAFLD on heart disease extends beyond the vascular disease to include cardiomyopathies, valvopathies, and arrhythmias ${ }^{[82]}$. NAFLD has been found to be a risk factor for the development of atrial fibrillation $(\mathrm{AF})^{[83,84]}$. In large cohort studies of patients with NAFLD with and without T2DM, NAFLD was found to be independently associated with $\mathrm{AF}^{[85,86]}$. A meta-analysis involving over 200,000 patients showed that NAFLD was associated with a two-fold increased risk of incidence of $\mathrm{AF}^{[87]}$. In addition, NAFLD has been shown to be independently associated with valvular disease, particularly aortic valve sclerosis and mitral annular calcification, which can subsequently lead to increased cardiovascular mortality and cardiac $\operatorname{arrhythmias}^{[8,89]}$.

Additionally, studies have linked NAFLD and diastolic dysfunction ${ }^{[0]}$. Compared to those without NAFLD, patients with NAFLD had alterations in cardiac remodeling, manifested by increased left ventricular mass index, left ventricular end-diastolic diameter, and left atrial volume index ${ }^{[00]}$. Both hepatic steatosis and fibrosis were significantly associated with increased left ventricular filling pressure, reflective of diastolic dysfunction, independent of other common cardiovascular risk factors ${ }^{[91]}$. In addition, those with NAFLD had impaired myocardial glucose uptake measured using fluorodeoxyglucose-positron emission tomography compared to those without $\mathrm{NAFLD}^{[92]}$. Further studies demonstrated that the severity of myocardial dysfunction was linearly correlated with the severity of biopsy-proven NAFLD. Interestingly, it was noted that myocardial alterations occurred prior to cirrhosis, suggesting that these changes may not be a consequence of portal hypertension ${ }^{[93,94]}$. The fibrosis-4 index, a marker of liver stiffness, was an independent predictor of left ventricular diastolic dysfunction, larger atrial volume, and higher all-cause mortality in patients with heart failure ${ }^{[95]}$. 
As demonstrated by multiple studies, there is a higher prevalence of clinically significant cardiac disease in patients with NAFLD than in the general population. This requires greater attention as their risk for CVD, including carotid and coronary atherosclerosis, appears to be increased unproportionally to traditional cardiometabolic risk factors, including metabolic syndrome and T2DM. Due to this elevated risk, this group of patients should undergo careful cardiovascular surveillance by screening for metabolic risk factors and intervening in modifiable risk factors.

\section{DIFFERENCES BETWEEN NAFLD AND MAFLD}

The above-presented data was gathered in patients diagnosed under the previously established NAFLD criteria. One would assume that patients diagnosed using the newly proposed MAFLD would show the same results; however, this may not entirely be the case. Recent studies have compared the characteristics of patients diagnosed by MAFLD and NAFLD criteria and found that the MAFLD population had higher liver enzymes and more glucose and lipid metabolism-related disorders ${ }^{[0,97]}$. MAFLD individuals had a lower GFR and higher prevalence of CKD compared to patients with NAFLD even after adjusting for sex, ethnicity, age, alcohol intake, and diabetes ${ }^{[98]}$. Patients with MAFLD were found to have a higher risk for cardiovascular adverse outcomes and a higher incidence of all-cause mortality compared to those with NAFLD $^{[97,99]}$. Furthermore, patients with concomitant MAFLD and viral hepatitis, but not NAFLD and viral hepatitis, had a significantly increased cardiovascular risk as assessed by cardiovascular disease risk estimator and atherogenic indices ${ }^{[100]}$. Tsutsumi et al ${ }^{[101]}$ recently used a generalized estimating equation approach to investigate the difference in atherosclerotic cardiovascular disease (ASCVD) risk between patients with MAFLD and NAFLD. Using this approach, they found that MAFLD better identifies patients with higher ASCVD risk, and this was due to the presence of metabolic dysfunction rather than moderate alcohol consumption. Another study showed that MAFLD was significantly associated with a higher risk of developing subclinical atherosclerosis as measured with carotid intima-media thickness and brachial-ankle pulse wave velocity ${ }^{[102]}$. A study of middle-aged Korean adults found that a considerable proportion of patients were found to have MAFLD without satisfying the former definition of NAFLD, and these individuals had a higher risk of CVD event ${ }^{[103]}$. Similar results have been reported from a community-based cohort study in suburban Sri Lanka ${ }^{[99]}$.

Fatty liver disease is a heterogenous disorder with different metabolic and genetic factors. Therefore, NAFLD diagnosis is based on the exclusion of other concomitant liver diseases, which does not accurately reflect the pathogenesis of this disease. On the other hand, MAFLD avoids the dichotomous view of nonalcoholic fatty liver and non-alcoholic steatohepatitis since it is based on positive criteria (see Table 1) instead of negative criteria ${ }^{[7]}$. Furthermore, this allows concomitant liver disease due to causes other than MAFLD and is called dual etiology, which was not possible under the previous definition of NAFLD ${ }^{[104]}$. For instance, a patient with a history of alcohol use disorder and criteria meeting MAFLD would have dual etiology of MAFLD and alcohol. Similarly, individuals with viral hepatitis and criteria meeting MAFLD would have dual etiology of MAFLD and viral hepatitis. Another subtype of MAFLD that merits discussion is patients with cirrhosis who fulfill the criteria for MAFLD. The proposed definition is MAFLD-related cirrhosis and to avoid the term cryptogenic cirrhosis ${ }^{[104]}$. The diagnostic criteria of MAFLD-related cirrhosis require a patient to have cirrhosis in the absence of typical histological signs suggestive of steatohepatitis with one of the following criteria: past or present metabolic risk factors, MAFLD on the previous biopsy, or previous documentation of steatosis by hepatic imaging.

As described above, MAFLD appears to better identify patients who are at higher risk of hepatic or cardiovascular outcomes. However, one drawback of this new definition is that it de-emphasizes the severity of hepatic steatosis, which has been shown to correlate with CVD outcomes and insulin resistance ${ }^{[105,106]}$. A 


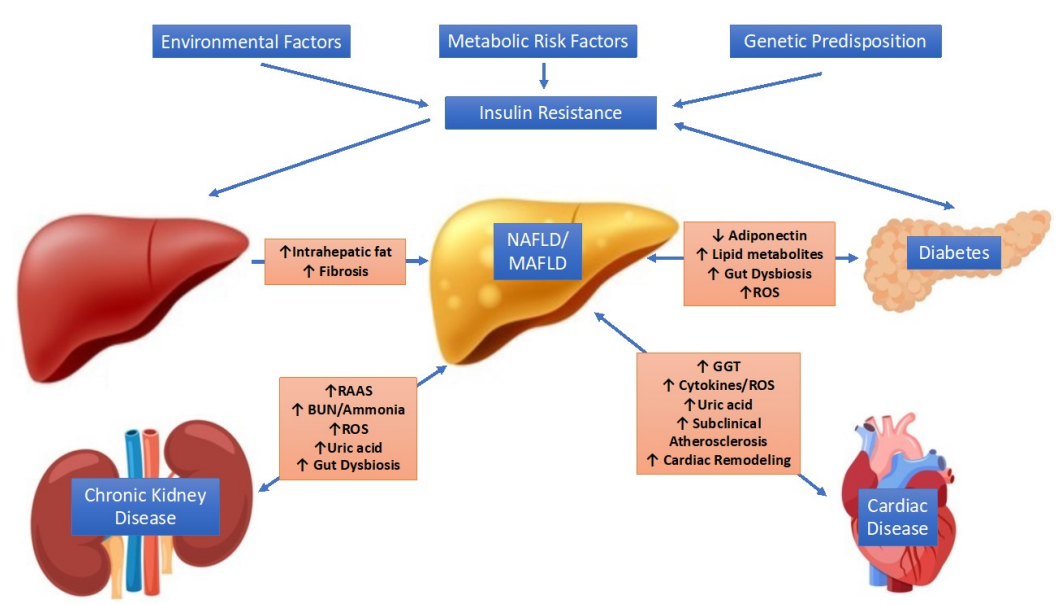

Figure 1. The proposed interplay between insulin resistance and NAFLD/MAFLD and subsequent development of diabetes, chronic kidney disease, and cardiac disease. ROS: Reactive oxygen species; BUN: blood urea nitrogen; RAAS: renin-angiotensin-aldosterone system; GGT: gamma-glutamyl transferase.

specific population of patients may be overlooked with this new definition, those with hepatitis steatosis and no metabolic risk factors, also previously known as lean NAFLD. A recent study using the NHANES III database compared patients with MAFLD and those with non-metabolic risk NAFLD and severe steatosis ${ }^{[107]}$. The cohort with non-metabolic risk NAFLD and severe steatosis was small, reflecting the rarity of this entity. This study showed that these patients had a similar degree of liver damage to those with MAFLD. Another study conducted in South Korea included patients with non-metabolic risk NAFLD, and while the proportion of subjects was too small to compare to patients with MAFLD, they found that patients with non-metabolic risk NALFD had an increased adjusted risk of CVD comparable to patients with MAFLD ${ }^{[102]}$. Interestingly, patients with non-metabolic risk NAFLD were significantly younger than those with MAFLD, which may suggest that these patients are being identified before overt signs of metabolic dysfunction ${ }^{[102,107]}$. These studies highlight that this is a population that may require close monitoring.

\section{CONCLUSION}

The evidence clearly shows that the clinical burden of NAFLD/MAFLD extends well beyond liver-related morbidity and mortality and is associated with multiple extrahepatic complications [Figure 1]. While most studies have been observational and retrospective, these associations require greater clinical awareness so that providers can provide multidisciplinary screening to patients with this illness. Moreover, using MAFLD criteria may be more practical for identifying patients with fatty liver disease with a high risk of disease progression. The main therapeutic interventions to manage these manifestations should include lifestyle and dietary modification as well as the early approach to pharmaceutical treatments, particularly with lipidlowering drugs and medications that augment insulin sensitivity. Future studies continue to be needed to better understand the mechanisms of these conditions and how we may potentially prevent them.

\section{DECLARATIONS}

\section{Authors' contributions}

Performed literature review and prepared the manuscript: Hassouneh R, Bhati C

Provided critical revisions: Hassouneh R, Siddiqui MS, Bhati C 


\section{Availability of data and material}

Not applicable.

\section{Financial support and sponsorship}

None.

\section{Conflicts of interest}

All authors declared that there are no conflicts of interest.

\section{Ethical approval and consent to participate}

Not applicable.

\section{Consent for publication}

Not applicable.

\section{Copyright}

(c) The Author(s) 2021.

\section{REFERENCES}

1. Chalasani N, Younossi Z, Lavine JE, et al. The diagnosis and management of nonalcoholic fatty liver disease: practice guidance from the American Association for the study of liver diseases. Hepatology 2018;67:328-57. DOI PubMed

2. Association for the Study of the Liver (EASL), European Association for the Study of Diabetes (EASD), European Association for the Study of Obesity (EASO). EASL-EASD-EASO Clinical Practice Guidelines for the management of non-alcoholic fatty liver disease. J Hepatol 2016;64:1388-402. DOI

3. Younossi Z, Anstee QM, Marietti M, et al. Global burden of NAFLD and NASH: trends, predictions, risk factors and prevention. Nat Rev Gastroenterol Hepatol 2018;15:11-20. DOI PubMed

4. Lazo M, Hernaez R, Eberhardt MS, et al. Prevalence of nonalcoholic fatty liver disease in the United States: the third national health and nutrition examination survey, 1988-1994. Am J Epidemiol 2013;178:38-45. DOI PubMed PMC

5. Masarone M, Federico A, Abenavoli L, Loguercio C, Persico M. Non alcoholic fatty liver: epidemiology and natural history. Rev Recent Clin Trials 2014;9:126-33. DOI PubMed

6. Anstee QM, Targher G, Day CP. Progression of NAFLD to diabetes mellitus, cardiovascular disease or cirrhosis. Nat Rev Gastroenterol Hepatol 2013;10:330-44. DOI PubMed

7. Eslam M, Sanyal AJ, George J; International Consensus Panel. MAFLD: a consensus-driven proposed nomenclature for metabolic associated fatty liver disease. Gastroenterology 2020;158:1999-2014.e1. DOI PubMed

8. Mantovani A, Byrne CD, Bonora E, Targher G. Nonalcoholic Fatty Liver Disease and Risk of Incident Type 2 Diabetes: A Metaanalysis. Diabetes Care 2018;41:372-82. DOI PubMed

9. Ekstedt M, Franzén LE, Mathiesen UL, et al. Long-term follow-up of patients with NAFLD and elevated liver enzymes. Hepatology 2006;44:865-73. DOI PubMed

10. Moschen AR, Wieser V, Tilg H. Adiponectin: key player in the adipose tissue-liver crosstalk. Curr Med Chem 2012;19:5467-73. DOI PubMed

11. Tilg H, Moschen AR. Adipocytokines: mediators linking adipose tissue, inflammation and immunity. Nat Rev Immunol 2006;6:77283. DOI PubMed

12. Zhang $\mathrm{H}, \mathrm{Niu} \mathrm{Y}, \mathrm{Gu} \mathrm{H}$, et al. Low serum adiponectin is a predictor of progressing to nonalcoholic fatty liver disease. J Clin Lab Anal 2019;33:e22709. DOI PubMed PMC

13. Khan RS, Bril F, Cusi K, Newsome PN. Modulation of Insulin Resistance in Nonalcoholic Fatty Liver Disease. Hepatology 2019;70:711-24. DOI PubMed

14. Chen CC, Sun YT, Chen JJ, Chang YJ. Tumor necrosis factor-alpha-induced cyclooxygenase-2 expression via sequential activation of ceramide-dependent mitogen-activated protein kinases, and IkappaB kinase 1/2 in human alveolar epithelial cells. Mol Pharmacol 2001;59:493-500. DOI PubMed

15. Pedersen HK, Gudmundsdottir V, Nielsen HB, et al; MetaHIT Consortium. Human gut microbes impact host serum metabolome and insulin sensitivity. Nature 2016;535:376-81. DOI PubMed

16. Boursier J, Mueller O, Barret M, et al. The severity of nonalcoholic fatty liver disease is associated with gut dysbiosis and shift in the metabolic function of the gut microbiota. Hepatology 2016;63:764-75. DOI PubMed PMC

17. Han R, Ma J, Li H. Mechanistic and therapeutic advances in non-alcoholic fatty liver disease by targeting the gut microbiota. Front Med 2018;12:645-57. DOI PubMed

18. Vrieze A, Van Nood E, Holleman F, et al. Transfer of intestinal microbiota from lean donors increases insulin sensitivity in individuals with metabolic syndrome. Gastroenterology 2012;143:913-6.e7. DOI PubMed 
19. Masarone M, Rosato V, Dallio M, et al. Role of oxidative stress in pathophysiology of nonalcoholic fatty liver disease. Oxid Med Cell Longev 2018;2018:1-14. DOI PubMed PMC

20. Patterson RE, Kalavalapalli S, Williams CM, et al. Lipotoxicity in steatohepatitis occurs despite an increase in tricarboxylic acid cycle activity. Am J Physiol Endocrinol Metab 2016;310:E484-94. DOI PubMed PMC

21. Angulo P, Kleiner DE, Dam-Larsen S, et al. Liver fibrosis, but no other histologic features, is associated with long-term outcomes of patients with nonalcoholic fatty liver disease. Gastroenterology 2015;149:389-97.e10. DOI PubMed PMC

22. Targher G, Bertolini L, Padovani R, et al. Prevalence of nonalcoholic fatty liver disease and its association with cardiovascular disease among type 2 diabetic patients. Diabetes Care 2007;30:1212-8. DOI PubMed

23. Targher G, Bertolini L, Rodella S, et al. Non-alcoholic fatty liver disease is independently associated with an increased prevalence of chronic kidney disease and proliferative/laser-treated retinopathy in type 2 diabetic patients. Diabetologia 2008;51:444-50. DOI PubMed

24. Targher G, Bertolini L, Chonchol M, et al. Non-alcoholic fatty liver disease is independently associated with an increased prevalence of chronic kidney disease and retinopathy in type 1 diabetic patients. Diabetologia 2010;53:1341-8. DOI PubMed

25. Williamson RM, Price JF, Glancy S, et al; Edinburgh Type 2 Diabetes Study Investigators. Prevalence of and risk factors for hepatic steatosis and nonalcoholic fatty liver disease in people with type 2 diabetes: the Edinburgh Type 2 Diabetes Study. Diabetes Care 2011;34:1139-44. DOI PubMed PMC

26. Zsóri G, Illés D, Ivány E, et al. In New-onset diabetes mellitus, metformin reduces fat accumulation in the liver, but not in the pancreas or pericardium. Metab Syndr Relat Disord 2019;17:289-95. DOI PubMed

27. Brandt A, Hernández-Arriaga A, Kehm R, et al. Metformin attenuates the onset of non-alcoholic fatty liver disease and affects intestinal microbiota and barrier in small intestine. Sci Rep 2019;9:6668. DOI PubMed PMC

28. Handzlik G, Holecki M, Kozaczka J, et al. Evaluation of metformin therapy using controlled attenuation parameter and transient elastography in patients with non-alcoholic fatty liver disease. Pharmacol Rep 2019;71:183-8. DOI PubMed

29. Musso G, Cassader M, Rosina F, Gambino R. Impact of current treatments on liver disease, glucose metabolism and cardiovascular risk in non-alcoholic fatty liver disease (NAFLD): a systematic review and meta-analysis of randomised trials. Diabetologia 2012;55:885-904. DOI PubMed

30. Sawangjit R, Chongmelaxme B, Phisalprapa P, et al. Comparative efficacy of interventions on nonalcoholic fatty liver disease (NAFLD): a PRISMA-compliant systematic review and network meta-analysis. Medicine (Baltimore) 2016;95:e4529. DOI PubMed PMC

31. He L, Liu X, Wang L, Yang Z. Thiazolidinediones for nonalcoholic steatohepatitis: A meta-analysis of randomized clinical trials. Medicine (Baltimore) 2016;95:e4947. DOI PubMed PMC

32. Said A, Akhter A. Meta-Analysis of Randomized Controlled Trials of Pharmacologic Agents in Non-alcoholic Steatohepatitis. Ann Hepatol 2017;16:538-47. DOI PubMed

33. Armstrong MJ, Gaunt P, Aithal GP, et al. Liraglutide safety and efficacy in patients with non-alcoholic steatohepatitis (LEAN): a multicentre, double-blind, randomised, placebo-controlled phase 2 study. Lancet 2016;387:679-90. DOI PubMed

34. Newsome PN, Buchholtz K, Cusi K, et al; NN9931-4296 Investigators. A Placebo-controlled trial of subcutaneous semaglutide in nonalcoholic steatohepatitis. N Engl J Med 2021;384:1113-24. DOI PubMed

35. Mantovani A, Petracca G, Beatrice G, Csermely A, Lonardo A, Targher G. Glucagon-like Peptide-1 receptor agonists for treatment of nonalcoholic fatty liver disease and nonalcoholic steatohepatitis: an updated meta-analysis of randomized controlled trials. Metabolites 2021;11:73. DOI PubMed PMC

36. Jia G, Di F, Wang Q, et al. Non-alcoholic fatty liver disease is a risk factor for the development of diabetic nephropathy in patients with Type 2 diabetes mellitus. PLoS One 2015;10:e142808. DOI PubMed PMC

37. Musso G, Gambino R, Tabibian JH, et al. Association of non-alcoholic fatty liver disease with chronic kidney disease: a systematic review and meta-analysis. PLoS Med 2014;11:e1001680. DOI PubMed PMC

38. Singal AK, Hasanin M, Kaif M, Wiesner R, Kuo YF. Nonalcoholic steatohepatitis is the most rapidly growing indication for simultaneous liver kidney transplantation in the United States. Transplantation 2016;100:607-12. DOI

39. Molnar MZ, Joglekar K, Jiang Y, et al. Association of pre-transplant renal function with liver graft and patient survival after liver trans- plantation in patients with nonalcoholic steatohepatitis. Liver Transplant 2019;25:399-410. DOI

40. Singal AK, Hasanin M, Kaif M, Wiesner RW, Kuo YF. MELD stratified outcomes among recipients with diabetes or hypertension: simulta- neous liver kidney versus liver alone. J Clin Gastroenterol 2018;52:67-72. DOI PubMed

41. Vilar-Gomez E, Calzadilla-Bertot L, Friedman SL, et al. Improvement in liver histology due to lifestyle modification is independently associated with improved kidney function in patients with non-alcoholic steatohepatitis. Aliment Pharmacol Ther 2017;45:332-44. DOI PubMed

42. Musso G, Cassader M, Cohney S, Pinach S, Saba F, Gambino R. Emerging liver-kidney interactions in nonalcoholic fatty liver disease. Trends Mol Med 2015;21:645-62. DOI PubMed

43. Mizuiri S, Ohashi Y. ACE and ACE2 in kidney disease. World J Nephrol 2015;4:74-82. DOI PubMed PMC

44. Morris E, Fletcher JA, Thyfault JP, Rector RS. The role of angiotensin II in nonalcoholic steatohepatitis. Mol Cell Endocrinol 2013;378:29-40. DOI PubMed

45. de Vries APJ, Ruggenenti P, Ruan XZ, et al. Fatty kidney: emerging role of ectopic lipid in obesity-related renal disease. Lancet Diabetes Endocrinol 2014;2:417-26. DOI PubMed

46. Osterreicher CH, Taura K, De Minicis S, et al. Angiotensin-converting-enzyme 2 inhibits liver fibrosis in mice. Hepatology 2009;50:929-38. DOI PubMed PMC 
47. Cheungpasitporn W, Thongprayoon C, O'Corragain OA, Edmonds PJ, Kittanamongkolchai W, Erickson SB. Associations of sugarsweetened and artificially sweetened soda with chronic kidney disease: a systematic review and meta-analysis. Nephrology (Carlton) 2014;19:791-7. DOI PubMed

48. Abdelmalek MF, Suzuki A, Guy C, et al; Nonalcoholic Steatohepatitis Clinical Research Network. Increased fructose consumption is associated with fibrosis severity in patients with nonalcoholic fatty liver disease. Hepatology 2010;51:1961-71. DOI PubMed PMC

49. Johnson RJ, Nakagawa T, Sanchez-Lozada LG, et al. Sugar, uric acid, and the etiology of diabetes and obesity. Diabetes 2013;62:3307-15. DOI PubMed PMC

50. Liabeuf S, Barreto DV, Barreto FC, et al; European Uraemic Toxin Work Group (EUTox). Free p-cresylsulphate is a predictor of mortality in patients at different stages of chronic kidney disease. Nephrol Dial Transplant 2010;25:1183-91. DOI PubMed

51. Wu IW, Hsu KH, Lee CC, et al. p-Cresyl sulphate and indoxyl sulphate predict progression of chronic kidney disease. Nephrol Dial Transplant 2011;26:938-47. DOI PubMed PMC

52. Vaziri ND, Yuan J, Khazaeli M, Masuda Y, Ichii H, Liu S. Oral activated charcoal adsorbent (AST-120) ameliorates chronic kidney disease-induced intestinal epithelial barrier disruption. Am J Nephrol 2013;37:518-25. DOI PubMed PMC

53. Federico A, Dallio M, Masarone M, Persico M, Loguercio C. The epidemiology of non-alcoholic fatty liver disease and its connection with cardiovascular disease: role of endothelial dysfunction. Eur Rev Med Pharmacol Sci 2016;20:4731-41. PubMed

54. Hamaguchi M, Kojima T, Takeda N, et al. Nonalcoholic fatty liver disease is a novel predictor of cardiovascular disease. World $J$ Gastroenterol 2007;13:1579-84. DOI PubMed PMC

55. Stepanova M, Younossi ZM. Independent association between nonalcoholic fatty liver disease and cardiovascular disease in the US population. Clin Gastroenterol Hepatol 2012;10:646-50. DOI PubMed

56. Sirota JC, McFann K, Targher G, Johnson RJ, Chonchol M, Jalal DI. Elevated serum uric acid levels are associated with nonalcoholic fatty liver disease independently of metabolic syndrome features in the United States: Liver ultrasound data from the National Health and Nutrition Examination Survey. Metabolism 2013;62:392-9. DOI PubMed PMC

57. Northup PG, Argo CK, Shah N, Caldwell SH. Hypercoagulation and thrombophilia in nonalcoholic fatty liver disease: mechanisms, human evidence, therapeutic implications, and preventive implications. Semin Liver Dis 2012;32:39-48. DOI PubMed

58. Targher G. Relationship between high-sensitivity C-reactive protein levels and liver histology in subjects with non-alcoholic fatty liver disease. J Hepatol 2006;45:879-81; author reply 881. DOI PubMed

59. Oni ET, Agatston AS, Blaha MJ, et al. A systematic review: burden and severity of subclinical cardiovascular disease among those with nonalcoholic fatty liver; should we care? Atherosclerosis 2013;230:258-67. DOI PubMed

60. Sookoian S, Pirola CJ. Non-alcoholic fatty liver disease is strongly associated with carotid atherosclerosis: a systematic review. $J$ Hepatol 2008;49:600-7. DOI PubMed

61. Zheng J, Zhou Y, Zhang K, et al. Association between nonalcoholic fatty liver disease and subclinical atherosclerosis: a crosssectional study on population over 40 years old. BMC Cardiovasc Disord 2018;18:147. DOI PubMed PMC

62. Brea A, Mosquera D, Martín E, Arizti A, Cordero JL, Ros E. Nonalcoholic fatty liver disease is associated with carotid atherosclerosis: a case-control study. Arterioscler Thromb Vasc Biol 2005;25:1045-50. DOI PubMed

63. Fracanzani AL, Burdick L, Raselli S, et al. Carotid artery intima-media thickness in nonalcoholic fatty liver disease. Am J Med 2008;121:72-8. DOI PubMed

64. Cai J, Zhang S, Huang W. Association between nonalcoholic fatty liver disease and carotid atherosclerosis: a meta-analysis. Int J Clin Exp Med 2015;8:7673-8. PubMed PMC

65. Chung GE, Choi SY, Kim D, et al. Nonalcoholic fatty liver disease as a risk factor of arterial stiffness measured by the cardioankle vascular index. Medicine (Baltimore) 2015;94:e654. DOI PubMed PMC

66. Salvi P, Ruffini R, Agnoletti D, et al. Increased arterial stiffness in nonalcoholic fatty liver disease: the Cardio-GOOSE study. $J$ Hypertens 2010;28:1699-707. DOI PubMed

67. Lee YJ, Shim JY, Moon BS, et al. The relationship between arterial stiffness and nonalcoholic fatty liver disease. Dig Dis Sci 2012;57:196-203. DOI PubMed

68. Gepner AD, Young R, Delaney JA, et al. Comparison of coronary artery calcium presence, carotid plaque presence, and carotid intima-media thickness for cardiovascular disease prediction in the Multi-Ethnic Study of Atherosclerosis. Circ Cardiovasc Imaging 2015;8:e02262. DOI PubMed PMC

69. Kim D, Choi SY, Park EH, et al. Nonalcoholic fatty liver disease is associated with coronary artery calcification. Hepatology 2012;56:605-13. DOI PubMed PMC

70. Park HE, Kwak MS, Kim D, Kim MK, Cha MJ, Choi SY. Nonalcoholic fatty liver disease is associated with coronary artery calcification development: a longitudinal study. J Clin Endocrinol Metab 2016;101:3134-43. DOI PubMed

71. Wong VW, Wong GL, Yip GW, et al. Coronary artery disease and cardiovascular outcomes in patients with non-alcoholic fatty liver disease. Gut 2011;60:1721-7. DOI PubMed

72. Choi SY, Kim D, Kim HJ, et al. The relation between non-alcoholic fatty liver disease and the risk of coronary heart disease in Koreans. Am J Gastroenterol 2009;104:1953-60. DOI PubMed

73. Targher G, Byrne CD, Lonardo A, Zoppini G, Barbui C. Non-alcoholic fatty liver disease and risk of incident cardiovascular disease: a meta-analysis. J Hepatol 2016;65:589-600. DOI PubMed

74. Ekstedt M, Hagström H, Nasr P, et al. Fibrosis stage is the strongest predictor for disease-specific mortality in NAFLD after up to 33 years of follow-up. Hepatology 2015;61:1547-54. DOI PubMed

75. Vilar-Gomez E, Calzadilla-Bertot L, Wai-Sun Wong V, et al. Fibrosis severity as a determinant of cause-specific mortality in patients with advanced nonalcoholic fatty liver disease: a multi-national cohort study. Gastroenterology 2018;155:443-457.e17. DOI 
PubMed

76. VanWagner LB, Lapin B, Skaro AI, Lloyd-Jones DM, Rinella ME. Impact of renal impairment on cardiovascular disease mortality after liver transplantation for nonalcoholic steatohepatitis cirrhosis. Liver Int 2015;35:2575-83. DOI PubMed PMC

77. Vanwagner LB, Bhave M, Te HS, Feinglass J, Alvarez L, Rinella ME. Patients transplanted for nonalcoholic steatohepatitis are at increased risk for postoperative cardiovascular events. Hepatology 2012;56:1741-50. DOI PubMed

78. Albeldawi M, Aggarwal A, Madhwal S, et al. Cumulative risk of cardiovascular events after orthotopic liver transplantation. Liver Transpl 2012;18:370-5. DOI PubMed

79. Wannamethee SG, Lennon L, Shaper AG. The value of gamma-glutamyltransferase in cardiovascular risk prediction in men without diagnosed cardiovascular disease or diabetes. Atherosclerosis 2008;201:168-75. DOI PubMed

80. Franzini M, Corti A, Martinelli B, et al. Gamma-glutamyltransferase activity in human atherosclerotic plaques--biochemical similarities with the circulating enzyme. Atherosclerosis 2009;202:119-27. DOI PubMed

81. Koenig G, Seneff S. Gamma-Glutamyltransferase: a predictive biomarker of cellular antioxidant inadequacy and disease risk. Dis Markers 2015;2015:818570. DOI PubMed PMC

82. Anstee QM, Mantovani A, Tilg H, Targher G. Risk of cardiomyopathy and cardiac arrhythmias in patients with nonalcoholic fatty liver disease. Nat Rev Gastroenterol Hepatol 2018;15:425-39. DOI PubMed

83. Alonso A, Misialek JR, Amiin MA, et al. Circulating levels of liver enzymes and incidence of atrial fibrillation: the Atherosclerosis Risk in Communities cohort. Heart 2014;100:1511-6. DOI PubMed PMC

84. Sinner MF, Wang N, Fox CS, et al. Relation of circulating liver transaminase concentrations to risk of new-onset atrial fibrillation. Am J Cardiol 2013;111:219-24. DOI PubMed PMC

85. Targher G, Valbusa F, Bonapace S, et al. Non-alcoholic fatty liver disease is associated with an increased incidence of atrial fibrillation in patients with type 2 diabetes. PLoS One 2013;8:e57183. DOI PubMed PMC

86. Targher G, Mantovani A, Pichiri I, et al. Non-alcoholic fatty liver disease is associated with an increased prevalence of atrial fibrillation in hospitalized patients with type 2 diabetes. Clin Sci (Lond) 2013;125:301-9. DOI PubMed

87. Wijarnpreecha K, Boonpheng B, Thongprayoon C, Jaruvongvanich V, Ungprasert P. The association between non-alcoholic fatty liver disease and atrial fibrillation: A meta-analysis. Clin Res Hepatol Gastroenterol 2017;41:525-32. DOI PubMed

88. Mantovani A, Pernigo M, Bergamini C, et al. Heart valve calcification in patients with type 2 diabetes and nonalcoholic fatty liver disease. Metabolism 2015;64:879-87. DOI PubMed

89. Markus MR, Baumeister SE, Stritzke J, et al. Hepatic steatosis is associated with aortic valve sclerosis in the general population: the Study of Health in Pomerania (SHIP). Arterioscler Thromb Vasc Biol 2013;33:1690-5. DOI PubMed

90. VanWagner LB, Wilcox JE, Colangelo LA, et al. Association of nonalcoholic fatty liver disease with subclinical myocardial remodeling and dysfunction: a population-based study. Hepatology 2015;62:773-83. DOI PubMed PMC

91. Azzam H, Malnick S. Non-alcoholic fatty liver disease - the heart of the matter. World J Hepatol 2015;7:1369-76. DOI PubMed PMC

92. Lee YH, Kim KJ, Yoo ME, et al. Association of non-alcoholic steatohepatitis with subclinical myocardial dysfunction in noncirrhotic patients. $J$ Hepatol 2018;68:764-72. DOI PubMed

93. Petta S, Argano C, Colomba D, et al. Epicardial fat, cardiac geometry and cardiac function in patients with non-alcoholic fatty liver disease: association with the severity of liver disease. J Hepatol 2015;62:928-33. DOI PubMed

94. Simon TG, Bamira DG, Chung RT, Weiner RB, Corey KE. Nonalcoholic steatohepatitis is associated with cardiac remodeling and dysfunction. Obesity (Silver Spring) 2017;25:1313-6. DOI PubMed PMC

95. Sato Y, Yoshihisa A, Kanno Y, et al. Liver stiffness assessed by Fibrosis-4 index predicts mortality in patients with heart failure. Open Heart 2017;4:e000598. DOI PubMed PMC

96. Lin S, Huang J, Wang M, et al. Comparison of MAFLD and NAFLD diagnostic criteria in real world. Liver Int 2020;40:2082-9. DOI PubMed

97. Nguyen VH, Le MH, Cheung RC, Nguyen MH. Differential clinical characteristics and mortality outcomes in persons with NAFLD and/or MAFLD. Clin Gastroenterol Hepatol 2021;S1542-3565(21)00567. DOI PubMed

98. Sun DQ, Jin Y, Wang TY, et al. MAFLD and risk of CKD. Metabolism 2021;115:154433. DOI PubMed

99. Niriella MA, Ediriweera DS, Kasturiratne A, et al. Outcomes of NAFLD and MAFLD: Results from a community-based, prospective cohort study. PLoS One 2021;16:e245762. DOI PubMed PMC

100. Guerreiro GTS, Longo L, Fonseca MA, de Souza VEG, Álvares-da-Silva MR. Does the risk of cardiovascular events differ between biopsy-proven NAFLD and MAFLD? Hepatol Int 2021;15:380-91. DOI PubMed

101. Tsutsumi T, Eslam M, Kawaguchi T, et al. MAFLD better predicts the progression of atherosclerotic cardiovascular risk than NAFLD: Generalized estimating equation approach. Hepatol Res 2021. DOI PubMed

102. Liu S, Wang J, Wu S, et al. The progression and regression of metabolic dysfunction-associated fatty liver disease are associated with the development of subclinical atherosclerosis: a prospective analysis. Metabolism 2021;120:154779. DOI PubMed

103. Lee H, Lee YH, Kim SU, Kim HC. Metabolic dysfunction-associated fatty liver disease and incident cardiovascular disease risk: a nationwide cohort study. Clin Gastroenterol Hepatol 2020;S1542-3565(20)31717. DOI PubMed

104. Eslam M, Newsome PN, Sarin SK, et al. A new definition for metabolic dysfunction-associated fatty liver disease: an international expert consensus statement. J Hepatol 2020;73:202-9. DOI PubMed

105. Brunner KT, Pedley A, Massaro JM, Hoffmann U, Benjamin EJ, Long MT. Increasing liver fat is associated with progression of cardiovascular risk factors. Liver Int 2020;40:1339-43. DOI PubMed

106. Mantovani A, Petracca G, Beatrice G, Tilg H, Byrne CD, Targher G. Non-alcoholic fatty liver disease and risk of incident diabetes 
mellitus: an updated meta-analysis of 501022 adult individuals. Gut 2021;70:962-9. DOI PubMed

107. Huang J, Kumar R, Wang M, Zhu Y, Lin S. MAFLD criteria overlooks a number of patients with severe steatosis: Is it clinically relevant? J Hepatol 2020;73:1265-7. DOI PubMed 